\title{
Sobre la evaluación del profesorado universitario (especial referencia a ciencias económicas y jurídicas)
}

\author{
Francisco J. Delgado*, Roberto Fernández-Llera*
}

Resumen: La evaluación del profesorado universitario ha avanzado notablemente desde la aprobación y el desarrollo de la LOU. En este trabajo ofrecemos una panorámica crítica del complejo entramado de la evaluación, con especial atención a la investigación y a los campos académicos de Economía y Derecho. Asimismo, se sugieren algunas propuestas de mejora para aumentar la eficiencia y la transparencia del sistema. La evaluación del profesorado resulta fundamental para mejorar la calidad del Sistema Universitario Español. También es muy pertinente para discriminar dentro del colectivo, en un avance hacia la carrera horizontal, mediante la medición de la productividad, tema polémico en el Sector Público, pero no irresoluble. Los datos muestran que, tras los primeros años de andadura de las evaluaciones, se impone una profunda reflexión para mejorar el sistema. En particular, se sugiere reducir la «burocracia» e incrementar la transparencia y la objetividad del sistema.

Palabras clave: Evaluación, universidad, indicadores bibliométricos, Economía, Derecho.

\section{On the evaluation of university lecturers (with special reference to Economics and Law)}

\begin{abstract}
The evaluation of university teaching staff has advanced notably since the approval and development of the LOU (Law of Universities). In this paper we present a critical panorama of the complex system of evaluation, with special attention to research and to the academic fields of Economics and Law. Furthermore, improvements are suggested for increasing the efficiency and transparency of the system. The evaluation of faculty members is fundamental for improving the quality of the Spanish University System. It is also pertinent for making distinctions among members of the community, in an approach towards horizontal promotion through productivity measurements. While this is a polemic topic in the public sector, it is not insurmountable. Data reveal that after the evaluations of the initial years, a serious reflection has begun for improving the system. Specifically, there are suggestions for reducing 'red tape' and increasing the transparency and neutrality of the system.
\end{abstract}

Keywords: evaluation, university, bibliometric indicators, Economics, Law.

Agradecemos a dos evaluadores anónimos de la revista los valiosos comentarios y sugerencias realizados al texto original que han contribuido significativa-

* Departamento de Economía. Universidad de Oviedo. Correo-e: fdelgado@uniovi.es, rfllera@uniovi.es.

Recibido: 10-05-2011; 2. ${ }^{a}$ versión: 06-09-2011; 3. ${ }^{a}$ versión: 03-01-2012; aceptado: 27-02-2012. 
mente a su mejora. Asimismo se agradecen los comentarios recibidos en el «VIII Foro sobre la Evaluación de la Calidad de la Investigación y de la Educación Superior» celebrado en Santander (junio de 2011). Los autores agradecen la financiación del proyecto MICINN-09-ECO2009-13864-C03-03.

\section{Introducción}

La mejora del sistema de educación superior se ha convertido en un aspecto crucial en los últimos años en nuestro país en sintonía con otras naciones en el contexto del proceso de Bolonia. La elaboración de diferentes clasificaciones o rankings de las universidades ${ }^{1}$ actúan asimismo como un incentivo a la permanente evaluación y mejora, en un contexto de mayor transparencia y rendición de cuentas por parte de las universidades.

En esa senda uno de los retos más destacados pasa por la necesidad (y conveniencia) de evaluar la actividad del profesorado universitario como parte sustancial del sistema. Tal evaluación adquiere diferentes finalidades, ya sea el acceso o promoción como también otros objetivos, por ejemplo los complementos retributivos tanto nacionales como autonómicos, que contribuyan a mejorar la universidad en su camino hacia la excelencia ${ }^{2}$.

En desarrollo de la LOU, en 2002 fue creada la fundación estatal ANECA como entidad básica de evaluación de la calidad de las universidades, tanto en lo referente a los títulos como a los profesores. Así, se han desarrollado diversos programas: PEP, programa de evaluación del profesorado laboral; ACADEMIA: programa de evaluación del profesorado funcionario; DOCENTIA: programa de evaluación de la actividad docente; entre otros.

Asimismo, algunas regiones han creado sus propias agencias autonómicas de evaluación, con competencias en el marco del profesorado laboral y de los complementos regionales. En la actualidad existen agencias propias en Andalucía, Aragón, Canarias, Castilla y León, Cataluña, Madrid, Comunidad Valenciana, Galicia, Islas Baleares y País Vasco.

Adicionalmente, la experiencia de evaluación al profesorado universitario nació con la CNEAI -Comisión Nacional de Evaluación de la Actividad Investigadora- al hilo del complemento de productividad o sexenios de investigación, allá por 1989.

Aunque con otros objetivos, no podemos dejar de señalar la existencia de la ANEP - Agencia Nacional de Evaluación y Prospectiva- creada en 1986 para evaluar la financiación de proyectos de investigación y otras ayudas a la I+D+i.

Otro aspecto relevante es el Estatuto del PDI, actualmente en borrador. En esta norma se contempla la carrera horizontal en el profesorado universitario, con el establecimiento de tres grados de ascenso dentro de los cuerpos de TU y CU, así como un cuarto grado de progresión, con un alto nivel de exigencia. Se contempla la evaluación académica global, voluntaria, cada 5 años según diversos criterios: antigüedad en el empleo público docente universitario, méritos docentes, 
méritos de investigación, méritos por innovación y transferencia de conocimiento, méritos de dirección y gestión académica universitaria, formación. Se pretende llevar a cabo por evaluación externa (por determinar) y permanece la evaluación específica de la actividad investigadora cada 6 años en los denominados sexenios.

Sin duda, el elemento que ha quedado hasta la fecha al margen del rigor de la evaluación es el referente a la actividad docente, ya que los períodos de 5 años o quinquenios son, a menudo, reconocidos de forma automática por las universidades.

En este trabajo pretendemos realizar una revisión crítica del sistema de evaluación del profesorado universitario con especial atención a las ramas de la economía y el derecho. La sección segunda sitúa al lector en el complejo mapa de la evaluación de los profesores de universidad, con mayor detenimiento en la vertiente de la investigación por su preponderancia en los resultados de la evaluación. En la sección tercera se revisan algunos resultados de los mecanismos de evaluación. Finalmente la sección cuarta incluye las conclusiones y consideraciones finales del trabajo.

\section{Las vertientes de la evaluación}

La evaluación del profesorado de las universidades españolas conforma un complejo entramado sintetizado en la figura 1. Por un lado, como evaluadores actúan diversos agentes: la agencia nacional (Aneca), las agencias autonómicas,

FIGURA 1

El complejo entramado de la evaluación

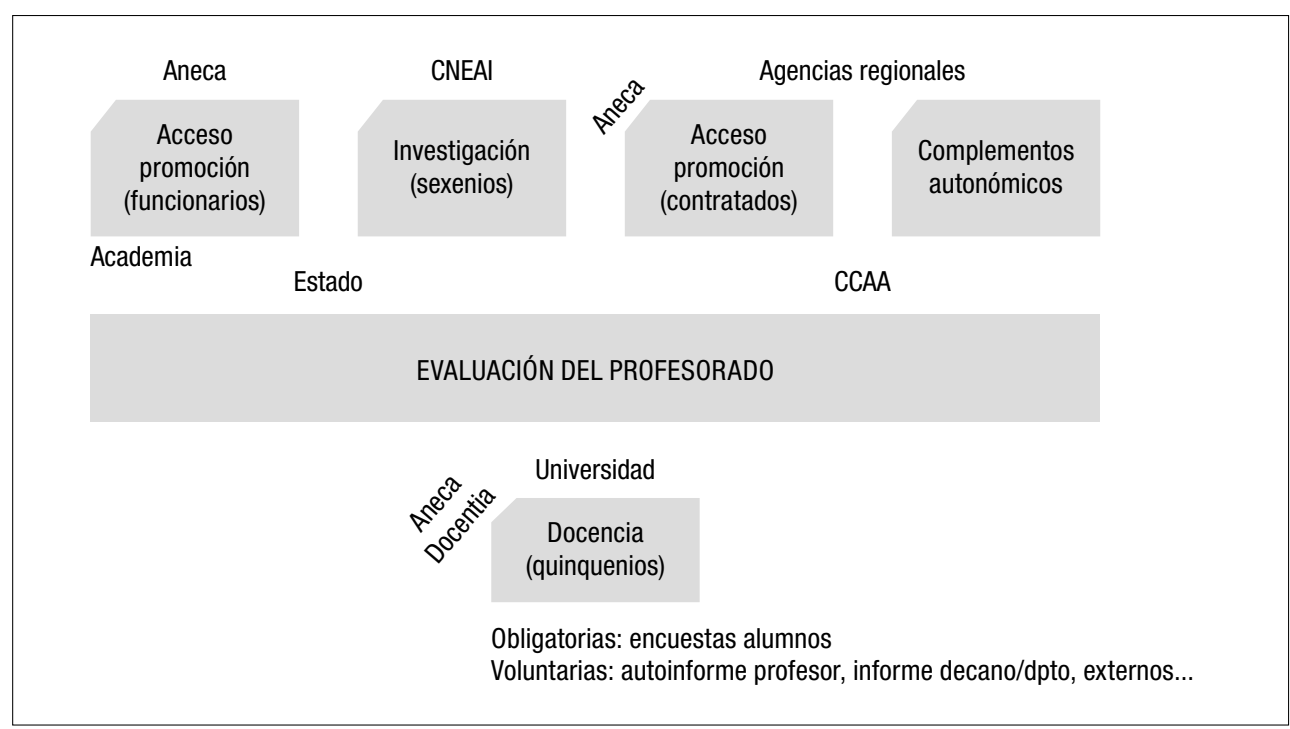

Fuente: elaboración propia. 
las propias universidades, la Comisión Nacional de Evaluación de la Actividad Investigadora (CNEAI). Por otro lado, se evalúa tanto el acceso y la promoción, como la investigación y la docencia. A la luz de esta diversidad surgen elementos de discusión y algunas necesidades que se pondrán de manifiesto en lo que resta de artículo, pero, en todo caso, sería positiva una mayor coordinación del conjunto del sistema de evaluación.

A continuación, realizaremos unos comentarios generales referidos a las grandes parcelas de la evaluación del profesorado: formación, docencia, investigación y gestión ${ }^{3}$. No obstante es positivo tener en cuenta los siguientes requisitos para acceder a las figuras más relevantes de profesorado. De acuerdo a la Aneca, en cuanto a la figura de Contratado Doctor, «se requiere una actividad investigadora intensa, desarrollada de forma continuada en el tiempo y centrada en unas líneas de investigación que permitan establecer cuál es su campo de investigación». Por otro lado, para Titular de Universidad, "se requiere una actividad investigadora intensa, de relevancia en su especialidad y que haya dado lugar a resultados reflejados en publicaciones, patentes, actividades de transferencia tecnológica o a trabajos que representen una innovación y avance en su campo, o que hayan tenido un impacto económico-social significativo. Además, se valora que se haya iniciado en actividades de liderazgo dentro de los trabajos realizados por varios autores, plasmadas en la dirección de tareas concretas en proyectos de investigación o contratos con empresas y organismos públicos». Finalmente, para Catedrático de Universidad, de manera similar al anterior aunque con mayor exigencia, «se requiere una actividad investigadora intensa, de calidad internacional en su especialidad y que haya dado lugar a resultados reflejados en publicaciones, patentes, actividades de transferencia tecnológica o a trabajos que representen una innovación y avance en su campo o que haya tenido un impacto económico-social significativo. Además, se considera necesario que el solicitante haya tenido un papel de liderazgo dentro de los trabajos realizados por varios autores, plasmado en la dirección de proyectos de investigación o contratos con empresas y organismos públicos». Véase más adelante la tabla V para la ponderación de los distintos bloques del currículo. Es evidente que un profesor universitario debe combinar docencia con investigación, pero es ciertamente complicado establecer ponderaciones adecuadas a estas parcelas según las diferentes categorías; no obstante, estamos de acuerdo con Pulido (2005) en que «confundir excelencia universitaria con excelencia investigadora es un desenfoque que todos debiéramos tratar de evitar», en referencia a la propuesta de un sector del colectivo que prima, a nuestro juicio excesivamente, la labor investigadora frente a la tarea docente del profesor.

\section{Formación}

Junto a la titulación exigida (doctor para las figuras analizadas en este trabajo), se valora tanto la formación pre-doctoral como la post-doctoral. Así, se ponderan méritos adicionales como las becas o los premios recibidos tales como los premios extraordinarios de licenciatura o doctorado. 


\section{Docencia}

Esta vertiente de la evaluación pasa por el análisis de la docencia impartida por el profesorado. Aun partiendo de la premisa de la dificultad (que no imposibilidad) de la evaluación de la docencia, se consideran aspectos como los quinquenios de docencia o los resultados de las encuestas realizadas a los alumnos. Adicionalmente se tienen en cuenta la dirección de tesis doctorales y trabajos de fin de máster o similares, la participación en proyectos de innovación docente, las publicaciones docentes tanto en libros como revistas, la asistencia a cursos y congresos orientados a la docencia, etc.

En este sentido consideramos un paso adelante la puesta en marcha del programa DOCENTIA aunque es necesario recorrer un largo camino en este campo. A modo de experiencia, García-Berro y otros (2010) describen el sistema de evaluación docente implantado en la Universidad Politécnica de Cataluña, modelo que incluye: $a$ ) autoinforme del profesor; $b$ ) planificación docente; $c$ ) opinión de los responsables académicos; $d$ ) opinión del alumnado; $e$ ) desarrollo de la actividad profesional. Por su parte, Tejedor (2009) aborda también el tópico de evaluar la docencia del profesor universitario y lleva a cabo una interesante disquisición sobre cómo debe ser un «buen profesor». A nuestro juicio la evaluación de la docencia debería ser obligatoria y con consecuencias, al igual que el resto de actividades del profesorado universitario, en aras a la eficiencia del sistema. Y para esa evaluación docente consideramos que ya se dispone de los cimientos adecuados para lanzar un procedimiento ágil que debería ser sencillo, objetivable y transparente, con el objetivo de medir o aproximar la calidad de las clases, la asistencia personalizada a los alumnos a través de las tutorías, las guías docentes, los materiales docentes, los portales virtuales propios, la participación en cursos y congresos de docencia, la innovación docente, etc. En este sentido se podría establecer siquiera una evaluación de mínimos. El nuevo Estatuto del PDI no parece ir en absoluto en esta dirección.

\section{Investigación}

La medición de la cantidad y calidad de la labor investigadora pasa por la necesidad de buscar indicios de calidad. Dejando a un lado las aportaciones a congresos y los proyectos de investigación, cuya valoración puede ser más objetiva, y los libros o capítulos de libros con algunas dificultades adicionales ${ }^{4}$, en el caso de los artículos en revistas, este tema se ha resuelto fundamentalmente con la creación de diferentes bases de datos o catálogos, algunos de ellos con cálculo del impacto de revistas.

A nivel mundial, nos encontramos con índices de impacto calculados en las dos bases de datos más relevantes, SCI y SSCI a través del Journal Citation Reports (JCR), y Scopus mediante los indicadores SJR5.

En el primer caso, el impacto JCR de un año (por ejemplo 2009) se determina dividiendo las citas recibidas en ese año (2009) por los trabajos publicados en los 
dos últimos años (2007 y 2008) entre el número de trabajos publicados en esos dos últimos (2007 y 2008). Adicionalmente, para evitar sesgos derivados del período de cálculo, también se computa el denominado "5-year impact» tomando como referencia los cinco años anteriores. A modo de ejemplo, en la tabla I se recogen las revistas españolas de los ámbitos de Economía y Empresa, y Derecho incluidas en SSCI. Aunque el número es reducido, es importante señalar la tendencia alcista en los últimos años, denotando el cambio experimentado. Otra observación importante es la necesaria comprobación de la lista actualizada de revistas en SSCI, ya que, por ejemplo, JCR 2009 contiene 245 revistas en la categoría "Economics» aunque hoy ya están 310 revistas admitidas en esa categoría en el SSCI aunque las nuevas sin impacto aún, como algunas de las recogidas en la tabla $\mathrm{I}^{6}$.

TABLA I

Revistas españolas Economía y Derecho. SSCI, SJR-Scopus y ANEP

\begin{tabular}{|c|c|c|c|}
\hline & \multirow{2}{*}{ a) SSCI } & \multirow{3}{*}{$\begin{array}{c}\text { b) SJR-Scopus } \\
\text { Incluida/ } \\
\text { Impacto }\end{array}$} & \multirow{3}{*}{$\begin{array}{c}\text { c) ANEP-CSIC } \\
\text { Categoría }\end{array}$} \\
\hline & & & \\
\hline Economía y Empresa & $\begin{array}{l}\text { Incluida/ } \\
\text { Impacto }\end{array}$ & & \\
\hline Hacienda Pública Española & Sí $\quad 0,375$ & Sí $\quad 0,025$ & A \\
\hline Investigaciones Económicas* & Sí $\quad 0,370$ & Sí $\quad 0,026$ & $\mathrm{~B}$ \\
\hline Revista de Economía Aplicada & Sí 0,125 & Sí $\quad 0,025$ & A \\
\hline Revista de Economía Mundial & Sí $\quad 0,032$ & Sí - & A \\
\hline Cuadernos de Economía y Dirección de la Empresa & Sí - & Sí - & $\mathrm{B}$ \\
\hline Revista de Historia Económica & Sí - & Sí $\quad 0,024$ & A+ \\
\hline Revista Española de Financiación y Contabilidad & Sí - & Sí $\quad 0,025$ & C \\
\hline Universia Business Review & Sí - & Sí $\quad 0,024$ & A \\
\hline Investigaciones Regionales & No & Sí $\quad 0,025$ & $\mathrm{C}$ \\
\hline $\begin{array}{l}\text { Revista de Métodos Cuantitativos para Economía } \\
\text { y Empresa }\end{array}$ & No & Sí $\quad 0,026$ & C \\
\hline Revista Galega de Economía & No & Sí $\quad 0,024$ & $\mathrm{C}$ \\
\hline $\begin{array}{l}\text { Applied Econometrics and International Develop- } \\
\text { ment }\end{array}$ & No & Sí - & $\mathrm{C}$ \\
\hline Estudios Económicos Regionales y Sectoriales & No & Sí $\quad 0,024$ & - \\
\hline Intangible capital & No & Sí - & C \\
\hline $\begin{array}{l}\text { Revista Europea de Dirección y Economía de la } \\
\text { Empresa }\end{array}$ & No & Sí - & $\mathrm{C}$ \\
\hline \multicolumn{4}{|l|}{ Derecho } \\
\hline Revista de Derecho Comunitario Europeo & Sí - & No & \\
\hline Revista Española de Derecho Constitucional & - & No & \\
\hline
\end{tabular}

Fuentes: ISI-JCR 2009, Scopus-SJR 2009, CSIC-ANEP (2008), DICE y elaboración propia.

* Investigaciones Económicas se ha fusionado en 2011 con Spanish Economic Review dando lugar a SERIES. 
En el caso de los indicadores SJR — Scimago Journal Rank- recogen la media ponderada de citas recibidas en un año (por ejemplo 2009) por los artículos publicados en los tres años anteriores (2006, 2007 y 2008) ${ }^{7}$ con el objetivo de analizar la influencia o prestigio de la revista.

En ambas bases de datos se pueden tomar las posiciones y los cuartiles como indicios de calidad de las revistas. Las revistas españolas con impacto se hallan por el momento en el último cuartil, fruto del escaso tiempo transcurrido desde su incorporación.

Además de la calidad de las revistas, la calidad de trabajos concretos puede medirse también por las citas recibidas. Las bases anteriores contienen las citas y en WOS-Web of Science puede consultarse Essential Science Indicators para saber, por ejemplo, cuántas citas ha de recibir un artículo para situarse entre el $1 \%, 5 \%$ o $10 \%$ más citados de la disciplina. Por supuesto Google Scholar es un buen complemento para la búsqueda de citas.

Como acabamos de ver, estas bases de datos resultan muy válidas para revistas «internacionales» (entendiendo las editadas fuera de España y, sobre todo, las editadas en lengua inglesa), pero no recogen demasiado bien la realidad de las revistas que se editan en nuestro país. Por este motivo se han desarrollado bases de datos con impactos para las revistas españolas: In-Recs ${ }^{8}$ para Ciencias Sociales, In-Recj’ para Ciencias Jurídicas. Con una filosofía similar a la de Thomson Reuters para los JCR, se dispone del índice de impacto anual y de los últimos 5 años. Además, esta herramienta puede ser útil también para citas a través de la opción «buscar por autores».

El impacto de un año (por ejemplo 2009) se computa por cociente entre las citas recibidas en ese año (2009) por los artículos publicados en los tres últimos años (2006, 2007 y 2008) entre el número de artículos de los tres últimos años (2006, 2007 y 2008).

Otra fuente de información relevante para las revistas españolas es la categorización realizada por la $\mathrm{ANEP}^{10}$. Así, se clasifican las revistas en cuatro categorías, de mayor a menor calidad: A, B, C y D. En la tabla I se recogen las revistas de economía y empresa y derecho catalogadas en las dos categorías más elevadas, A y B. Se puede observar que la presencia de las revistas en los catálogos internacionales con índices de impacto, especialmente SSCI-JCR, resulta clave para la clasificación de las revistas en el ámbito económico.

La base de datos DICE —Difusión y Calidad Editorial de las Revistas Españolas de Humanidades y Ciencias Sociales y Jurídicas - constituye una excelente herramienta para las revistas españolas. Esta base incluye múltiple información relativa a las bases de datos en las que están indexadas las revistas, y nuevos indicadores como la «valoración de la difusión internacional» o la «internacionalidad de las contribuciones» incorporados recientemente tras la revisión de DICE a comienzos de 2011. Es importante destacar que la ANECA utiliza esta base de datos como referencia de calidad de las publicaciones españolas en sus distintos procesos de evaluación de profesorado, tal y como se contempla en la propia web de DICE y en la documentación de la ANECA en sus programas PEP y ACADEMIA. 
Adicionalmente, existen otros catálogos de referencia aunque sin impactos, como el Catálogo Latindex y sus criterios, 33 para revistas impresas y 36 para revistas digitales. Para formar parte de ese catálogo, se deben reunir al menos 25 criterios. Como puede observarse en la tabla II, la principal limitación de Latindex es la ausencia de categorización, aunque se podrían tomar los criterios cumplidos como referencia, siempre con el requisito de evaluadores externos como condición necesaria para la calidad.

Otro sistema de categorización de revistas es CARHUS, desarrollado en Cataluña por la AGAUR —Agencia de Gestió d'Ajuts Universitaris i de Recerca- La última edición correspondiente a 2010 establece una clasificación para las revistas de mayor a menor calidad, en los grupos A, B, C, y D. Adicionalmente FECYT también lleva a cabo procesos de evaluación de la calidad de las revistas españolas (Coslado y otros, 2010).

TABLA II

Revistas españolas en Latindex. Economía y Derecho

\begin{tabular}{lr}
\hline Economía & 107 \\
Total & 25 \\
33 criterios & \\
\hline Derecho & 122 \\
Total & 9 \\
33 criterios & \\
\hline
\end{tabular}

Fuente: Latindex y DICE.

\section{Gestión e innovación}

En el campo de gestión se tienen en cuenta, fundamentalmente, los cargos académicos unipersonales desempeñados. También tiene relevancia, aunque menor, haber sido investigador principal de proyectos de investigación competitivos o haber desempeñado cargos representativos en órganos colegiados. En términos estrictos de innovación, apenas se ha desplegado un baremo común.

\section{Algunos resultados de las evaluaciones}

Con el ánimo de sintetizar la evaluación del profesorado laboral y funcionario, en la tabla III se recogen las puntuaciones de las diferentes parcelas objeto de análisis. Aunque no son estrictamente comparables, se observa la exigencia creciente de puntos en el baremo, desde los 55 del ayudante doctor o contratado doctor, hasta los 80 para el acceso a CU pasando por los 65 de TU.

En la tabla IV aparecen las cifras de evaluación positiva para 2009, año en el que ya existe cierta experiencia en el proceso para valorar los resultados alcan- 
TABLA III

Algunas claves de la evaluación del profesorado

\begin{tabular}{l|c|c|c|c}
\hline \multirow{2}{*}{ Apartados } & \multicolumn{4}{c}{ Puntuación máxima } \\
\cline { 2 - 5 } & $\begin{array}{c}\text { Ayudante } \\
\text { Doctor }\end{array}$ & $\begin{array}{c}\text { Contratado } \\
\text { Doctor }\end{array}$ & $\begin{array}{c}\text { Titular } \\
\text { Universidad }\end{array}$ & $\begin{array}{c}\text { Catedrático } \\
\text { Universidad }\end{array}$ \\
\hline 1. Actividad investigadora & 60 & 60 & 50 & 55 \\
\hline 2. Actividad docente & 35 & 30 & 40 & 35 \\
\hline 3. Formación docente & 5 & 8 & 5 & - \\
\hline 4. Otros (Gestión) & 100 & 100 & 5 & 10 \\
\hline Total & & 55 & 65 & 80 \\
\hline $\begin{array}{l}\text { Para evaluación positiva } \\
\text { Mínimo (total) } \\
\text { Submínimos }\end{array}$ & 55 & $50(1+2)$ & $60(1+2)$ & $20(2)$ \\
\hline
\end{tabular}

Fuente: elaboración propia a partir de ANECA.

\section{TABLA IV}

Cifras de los programas de evaluación del profesorado (2009). Porcentajes de evaluación positiva

\begin{tabular}{l|c|c|c}
\hline \multicolumn{1}{c}{ Comité } & Contratado Doctor & Ayudante Doctor & Colaborador \\
\hline Ciencias Sociales y Jurídicas & 55 & 69 & 73 \\
\hline Experimentales & 56 & 85 & 50 \\
\hline Salud & 55 & 80 & 86 \\
\hline Técnicas & 51 & 81 & 40 \\
\hline Humanidades & 49 & 75 & 50 \\
\hline Total & 54 & 78 & 68 \\
\hline
\end{tabular}

\begin{tabular}{l|c|c}
\hline \multicolumn{2}{c}{ b) Acreditación nacional } \\
\hline Ciencisión & TU & CU \\
\hline Ciencias & 47 & 61 \\
\hline Ciencias de la Salud & 74 & 81 \\
\hline Ingeniería y Arquitectura & 62 & 68 \\
\hline Arte y Humanidadicas & 66 & 67 \\
\hline Total & 74 & 65 \\
\hline
\end{tabular}

Fuente: Memoria de actividades 2009. ANECA. 
zados. En el ámbito de las figuras laborales, los datos aportan una evaluación positiva en el $54 \%$ de las solicitudes, porcentajes más elevados para las figuras menos exigentes, 78\% para ayudante doctor y 68\% para colaborador.

En el caso de las figuras funcionariales, las evaluaciones favorables suponen el $63 \%$ en los TU y el 69\% en los CU. Más allá de la cifra agregada cuya valoración es muy complicada por los múltiples factores que confluyen en ella, los datos ponen en evidencia una gran disparidad en los resultados por áreas de conocimiento. Sin lugar a dudas, el porcentaje más destacado es el escaso $47 \%$ de los TU en Ciencias Sociales y Jurídicas, 16 puntos por debajo del total, diferencia significativa que podría esconder diferentes grados de exigencia en los solicitantes. Ahondando un poco más en esta cuestión, estas reducidas tasas de éxito en este campo pueden evidenciar algunas lagunas propias de los solicitantes, como la tardía incorporación a las revistas internacionales de prestigio no demandadas en otras épocas y de súbita exigencia, así como también otras cuestiones específicas de estas áreas que posiblemente estén siendo minusvaloradas en los procesos de evaluación, como los artículos publicados en revistas «nacionales». Como recoge Pulido (2005), «el campo de las humanidades, ciencias sociales y jurídicas, en general, y de la economía, en particular, tiene algunas peculiaridades respecto a las ciencias experimentales, en particular en cuanto a canales y contenido de publicaciones, proyectos de investigación y contratos con empresas o Administraciones Públicas».

Por lo que respecta a los sexenios de investigación (tabla V) se aprecia nuevamente una gran disparidad de resultados por áreas de conocimiento. Como recogía San Segundo (2005) al reflejar los porcentajes de profesores "Sorteables» para las comisiones de las extintas habilitaciones nacionales, "en el conjunto de áreas económicas, existe una cierta evidencia de dureza relativa de las evaluaciones", ya que en Economía de forma agregada lo eran tan sólo el 29,9\% de los titulares y el 37,8\% de los catedráticos, frente 58,9\% y 64,6\% en el conjunto de campos. Sin embargo, en Derecho los porcentajes eran similares al promedio, con un $59,7 \%$ y 70,4\% respectivamente ${ }^{11}$. A modo de ejemplo hemos tomado cinco áreas y resulta llamativo el escaso éxito en las áreas de Economía Aplicada y Fundamentos del Análisis Económico. Así, en la primera, el 28,27\% de los TU no han solicitado aún sexenios y el $26,88 \%$ sí lo han solicitado pero les ha sido denegado. En suma, el 55,15\% de los TU no poseían ningún sexenio en 2009, ya sea por ausencia de solicitud o denegación. Si a esa cifra le añadimos el $32,03 \%$ de profesores con 1 sexenio, es posible concluir que el 87,18\% de los TU tenían, como máximo, un sexenio de investigación, con las consecuencias derivadas, si bien este porcentaje agregado debe tomarse con las debidas cautelas. En el resto de áreas analizadas estos porcentajes son notablemente inferiores. Estos resultados han provocado desde hace tiempo gran sensibilidad entre el colectivo y recientemente se ha lanzado una propuesta por parte de la Conferencia Española de Decanos de Economía y Empresa (tabla VI). A nuestro juicio, esta propuesta va en la dirección correcta por cuanto supone añadir transparencia y objetividad a la evaluación, aunque el contenido concreto de la misma es 
TABLA V

Algunos datos sobre la evaluación de sexenios de investigación (\%)

\begin{tabular}{l|c|r|r|r|r|r|r|r|r}
\hline \multicolumn{2}{c}{ Sexenios } & \multicolumn{1}{c|}{ NP } & $\mathbf{0}$ & $\mathbf{1}$ & $\mathbf{2}$ & $\mathbf{3}$ & $\mathbf{4}$ & $\mathbf{5}$ & $\mathbf{6}$ \\
\hline 225 & TU & 28,27 & 26,88 & 32,03 & 11,00 & 1,67 & 0,14 & 0,00 & 0,00 \\
Economía Aplicada & CU & 6,49 & 15,65 & 24,05 & 21,37 & 19,08 & 10,69 & 2,67 & 0,00 \\
\hline 415 & TU & 11,80 & 9,73 & 48,38 & 23,89 & 5,31 & 0,59 & 0,00 & 0,29 \\
Fund. Análisis Económico & CU & 6,84 & 8,55 & 8,55 & 17,09 & 28,21 & 18,80 & 9,40 & 2,56 \\
\hline 165 & TU & 9,09 & 16,04 & 44,92 & 25,67 & 2,67 & 1,60 & 0,00 & 0,00 \\
Derecho Mercantil & CU & 4,65 & 1,16 & 12,79 & 34,88 & 17,44 & 11,63 & 4,65 & 12,79 \\
\hline 140 & TU & 6,63 & 12,76 & 51,02 & 22,96 & 5,61 & 1,02 & 0,00 & 0,00 \\
Derecho del Trabajo & CU & 0,00 & 0,00 & 3,17 & 25,40 & 26,98 & 23,81 & 14,29 & 6,35 \\
\hline 750 & TU & 3,05 & 5,84 & 17,77 & 40,36 & 24,62 & 7,61 & 0,76 & 0,00 \\
Química Analítica & CU & 0,00 & 0,80 & 1,60 & 4,00 & 14,40 & 40,00 & 29,60 & 9,60 \\
\hline
\end{tabular}

Fuente: elaboración propia a partir de datos CNEAI 2009.

\section{TABLA VI}

Propuesta de la Conferencia Española de Decanos de Economía y Empresa para los sexenios de investigación

\begin{tabular}{l|c|c}
\hline \multicolumn{1}{c|}{ Tipo } & $\begin{array}{c}\text { Propuesta } \\
\text { Conferencia Decanos } \\
\text { Economía y Empresa }\end{array}$ & Propuesta propia \\
\hline Artículos JCR primer cuartil & 12 puntos & 12 puntos \\
\hline Artículos JCR segundo cuartil & 10 puntos & 10 puntos \\
\hline Artículos JCR tercer cuartil & 8 puntos & 8 puntos \\
Artículos In-Recs primer cuartil & 8 puntos & 5 puntos \\
Artículos Scopus & 8 puntos & 6 puntos \\
\hline Artículos JCR cuarto cuartil & 7 puntos & 4 puntos \\
Artículos In-Recs segundo cuartil & 7 puntos & 5 puntos \\
Artículos Econlit, IBSS, catálogo Latindex & 7 puntos & 3 puntos \\
\hline Artículos In-Recs tercer cuartil & - & 2 puntos \\
\hline Artículos In-Recs cuarto cuartil & - & - \\
\hline
\end{tabular}

Fuente: Conferencia Española de Decanos de Economía y Empresa y elaboración propia.

susceptible de discusión, siendo a nuestro juicio algo laxa. En la tabla aparece tanto la propuesta de la Conferencia como la de los autores del presente trabajo, coincidentes en el tratamiento de los artículos JCR, pero no en el resto. Entendemos que una propuesta similar debería figurar en los criterios para la evaluación de la investigación en los procesos de acreditación del profesorado universitario, adaptando las puntuaciones al baremo correspondiente. Una posibilidad sería proponer equivalencias entre las puntuaciones máximas de la evaluación 
investigadora con los sexenios de investigación, revisando al alza las actuales referencias sobre el reconocimiento de 15 puntos en la acreditación por cada sexenio reconocido.

\section{Conclusiones y consideraciones finales}

La generalización de la evaluación en el seno universitario debe tomarse como un gran avance hacia la mejora del sistema educativo español en un proceso de internacionalización y búsqueda de la excelencia en nuestra universidad.

En particular, la evaluación del profesorado universitario resulta necesaria para mejorar la calidad de la universidad española, y pertinente para discriminar en el colectivo en un avance hacia la carrera horizontal entroncada con la medición de la productividad, tema especialmente complicado en el ámbito público.

Sin embargo, como hemos visto, la evaluación actual resulta compleja y dispersa. El sistema actual resulta ineficiente y favorece la ingeniería curricular creativa o dispersión de la actividad del profesor universitario. Se requiere entonces diseñar un sistema objetivo, claro y transparente.

En cuanto a las comisiones de acreditación, debería recuperarse uno de los pocos aspectos positivos de las extintas habilitaciones nacionales, el sorteo para formar parte de la comisión, y los expertos no deberían ser anónimos, al menos en el caso de las evaluaciones negativas, tal y como recomienda el propio Consejo de Universidades (Comisión de Reclamaciones del Consejo de Universidades, Marzo 2009). También sería positivo lograr una mayor concordancia entre la especialización de los evaluadores y la del solicitante.

Si el sistema está basado en puntuaciones, sería deseable establecer valores para cada mérito o grupo de méritos, es decir, cuánto vale un artículo en cierta revista, un libro en determinada editorial, una aportación a un congreso, un proyecto de investigación, una tesis dirigida, una estancia, un año de cargo académico, etc., de forma que la aplicación determine un resultado inicial de la evaluación, a expensas de la validación de la comisión cuya experiencia debería servir para matizar ese resultado previo, siempre con la necesaria visión global, de conjunto, de la trayectoria académica evaluada.

También sería deseable revisar el papel de los «impactos» de las revistas: ¿qué relación existe con la calidad?, ¿existen indicadores alternativos?, ¿ranking o mejor categorización? Una excelente propuesta de debate ha sido aportada recientemente por Torres-Salinas y otros (2010). En cualquier caso, consideramos la bondad de asignar a priori puntuaciones mínimas a los artículos según las revistas (por cuartiles o terciles por ejemplo si hay impactos, por categorías si hay bloques) modulables al alza en función de las citas recibidas u otros indicios de calidad que se estimen oportunos.

En suma, tras estos años de andadura de las evaluaciones puede ser un buen momento para hacer una profunda reflexión y mejora del sistema, con un descenso de la "burocracia» y un incremento de la transparencia y objetivación de 
la evaluación. Junto a la experiencia de la ANECA como agente evaluador, se cuenta con aportaciones como Alegre (2007), Buela-Casal (2007), Buela-Casal y Sierra (2007), Murillo (2008), Escudero y otros (2010), que a buen seguro pueden contribuir al perfeccionamiento de la evaluación.

\section{Bibliografía}

Alegre Ávila, J. M. (2007). El nuevo sistema de selección del profesorado universitario funcionario. Revista Española de Derecho Administrativo, vol. 135, 437-457.

Buela-Casal, G. (2007). Reflexiones sobre el sistema de acreditación del profesorado funcionario de Universidad en España. Psicothema, vol. 19 (3), 473-482 (acceso: http:// dialnet.unirioja.es/servlet/articulo?codigo=2333649).

Buela-Casal, G.; Sierra, J. C. (2007). Criterios, indicadores y estándares para la acreditación de Profesores Titulares y Catedráticos de Universidad. Psicothema, vol. 19, 357-369 (acceso: http://dialnet.unirioja.es/servlet/articulo?codigo=2389417).

Coslado, M. A.; Báez, J. M.; Lacunza, I. (2010). Descripción y análisis del proceso de evaluación de la calidad de las revistas científicas españolas llevado a cabo por FECYT en el año 2008. Revista Española de Documentación Científica, vol. 33 (3), 481-495.

Escudero Escorza, T.; Pino Mejías, J. L.; Rodríguez Fernández, C. (2010). Evaluación del profesorado universitario para incentivos individuales. Revista de Educación, vol. 351, 513-537 (acceso: http://www.revistaeducacion.mec.es/re351/re351_21.pdf).

García-Berro, E.; Roca, S.; Amblás, G.; Murcia, F.; Sallarés, J.; Bugeda, G. (2010). La evaluación de la actividad docente del profesorado en el marco del EEES. Aula Abierta, vol. 38 (2), 29-40.

Gavel, Y.; Iselid, L. (2008). "Web of science and Scopus: A journal title overlap study». Online Information Review, vol. 32 (1), 8-21.

Murillo, F. J. (2008). La evaluación del profesorado universitario en España. Revista Iberoamericana de Evaluación Educativa, vol. 1 (3) (acceso: http://dialnet.unirioja.es/ servlet/articulo?codigo=2789110).

Pulido, A. (2005). Indicadores de calidad en la evaluación del profesorado universitario. Estudios de Economía Aplicada, vol. 23 (3), 667-684.

San Segundo, M. J. (2005). Promoción y remuneración del profesorado universitario: de la LRU a la LOU. Hacienda Pública Española, vol. 172, 93-117.

Tejedor, F. J. (2009). Evaluación del profesorado universitario: enfoque metodológico y algunas aportaciones de la investigación. Estudios sobre Educación, vol. 16, 79-102.

Torres-Salinas, D.; Jiménez-Contreras, E. (2010). Introducción y estudio comparativo de los nuevos indicadores de citación sobre revistas científicas en Journal Citation Reports y Scopus. El profesional de la información, vol. 19 (2), 201-207.

Torres-Salinas, D.; Bordons, M.; Giménez-Toledo, E.; Delgado-López, E.; Jiménez-Contreras, E.; Sanz-Casado, E. (2010). Clasificación integrada de revistas científicas (CIRC): propuesta de categorización de las revistas en ciencias sociales y humanas. El profesional de la información, vol. 19 (6), 675-683. 


\section{Enlaces}

ANECA [http://www.aneca.es].

CNEAI [http://www.educacion.es/cneai].

In-Recs [http://ec3.ugr.es/in-recs/].

In-Recj [http://ec3.ugr.es/in-recj/].

WOK - Web of Knowledge ISI-Thomson [acceso a través de FECYT].

Scopus [www.scopus.com] [http://www.scimagojr.com].

DICE [http://epuc.cchs.csic.es/dice].

CARHUS [http://www10.gencat.net/agaur_web/AppJava/castellano/a_info.jsp?contingut= carhus_2010].

\section{Notas}

[1] Existen diferentes rankings como el elaborado por la Universidad Jiao Tong de Shangai (http://www.arwu.org). Otras clasificaciones de prestigio son HIGHER, de The Times (http://www.timeshighereducation.co.uk/world-university-rankings/index. html), o el ranking Webometrics (http://www.webometrics.info/).

[2] El Campus de Excelencia Internacional «busca mejorar la calidad de nuestras universidades y conducir hasta la excelencia a los mejores campus en beneficio del conjunto de la sociedad, mediante la agregación, la especialización, la diferenciación y la internacionalización del sistema universitario español» (Fuente: http://www.educacion.es/campus-excelencia.html).

[3] San Segundo (2005) expone los cambios en la evaluación del profesorado consecuencia de la normativa LOU respecto a LRU.

[4] Existen criterios como la calidad de la editorial y la selección por evaluación de los libros, la posesión de ISBN, la colección en la que se publica, las citas recibidas, las reseñas en revistas de prestigio, la traducción de la obra a otros idiomas, etc. En cualquier caso la valoración de los libros es muy desigual por campos de conocimiento, siendo más importantes en el área de Derecho que en Económicas donde priman los artículos en revistas de prestigio con evaluación externa incluidos en SSCI.

[5] Véase Gavel y Iselid (2008) para una visión conjunta de ambas bases.

[6] La lista actualizada de Economics puede verse en: http://scientific.thomsonreuters. $\mathrm{com} /$ cgi-bin/jrnlst/jlresults.cgi?PC=SS\&SC $=$ GY.

[7] Otro indicador dentro del mismo proyecto es el SNIP - Source Normalized Impact per Paper (http://www.journalindicators.com). Asimismo Thomson Reuters también ofrece los denominados eigenfactor y article influence score (www.eigenfactor.org). Para más detalles sobre estos indicadores también puede consultarse el trabajo de Torres-Salinas y Jiménez-Contreras (2010).

[8] En In-RecS (http://ec3.ugr.es/in-recs/) se distinguen: Antropología, Biblioteconomía y Documentación, Ciencia Política y de la Administración, Comunicación, Economía, Educación, Geografía, Psicología, Sociología, Urbanismo.

[9] En In-RecJ (http://ec3.ugr.es/in-recj/) se consideran: Administrativo, Civil y Mercantil, Constitucional, Eclesiástico, Filosofía del Derecho, Multidisciplinar, Financiero y Tributario, Internacional, Penal y Procesal, Romano, Trabajo. 
[10] Se ha utilizado la clasificación de septiembre 2008 accesible a través del enlace: http:// www.ucm.es/centros/cont/descargas/documento15882.pdf. Recientemente se ha procedido a revisar este texto; si bien aún no está publicado, sí se pueden consultar los resultados a través de DICE aunque preferimos esperar a su publicación para su incorporación en este trabajo. Esta clasificación tuvo su origen en el grupo de trabajo establecido por la ANEP en el que se definieron los criterios de calidad para categorizar las revistas científicas españolas de Humanidades. No obstante, el desarrollo y la aplicación de los criterios fueron del Grupo de Investigación de Evaluación de Publicaciones Científicas del CCHS. CSIC y la categorización se actualiza de forma dinámica, mostrándose a través de DICE.

[11] La exigencia era de un sexenio para los titulares y dos para los catedráticos. 